(2) Open Access Full Text Article

\title{
An uncommon granulocytic sarcoma of the breast: a case report and literature review
}

This article was published in the following Dove Press journal:

OncoTargets and Therapy

\author{
Jie Zhai* \\ Xiangyi Kong* \\ Xue Yang* \\ Jidong Gao \\ Lixue Xuan \\ Xiang Wang \\ Jing Wang \\ Yi Fang
}

Department of Breast Surgical Oncology, National Cancer Center/ Cancer Hospital, Chinese Academy of Medical Sciences and Peking Union Medical College, Beijing, People's

Republic of China

*These authors contributed equally to this work
Correspondence: Jing Wang; Yi Fang Department of Breast Surgical Oncology, National Cancer Center/Cancer Hospital, Chinese Academy of Medical Sciences and Peking Union Medical College, Chaoyang District, Panjiayuan, Nanli 17, Beijing 100021, People's Republic of China

Emailwwwijj1234@vip.sina.com; fangyi050I@vip.sina.com

\begin{abstract}
Granulocytic sarcoma (GS) is an uncommon extramedullary manifestation of acute myeloid leukemia. GS is often likely to be clinically misdiagnosed as another type of primary breast cancer due to its rarity. We report an uncommon case of breast GS in a patient and review the relevant literature.
\end{abstract}

Keywords: granulocytic sarcoma, breast, misdiagnosis, immunohistochemistry

\section{Introduction}

Acute myeloid leukemia (AML) is generally considered to include a group of relatively well-defined cancers of the myeloid lineage of blood cells. In AML patients, immature leukocytes grow rapidly in the bone marrow and interfere with the production of normal blood cells. Granulocytic sarcoma (GS) is an uncommon extramedullary manifestation of AML and refers to an extramedullary mass consisting of myeloid blasts with or without maturation, which destroys the normal tissue architecture. Although myeloid sarcoma has been listed in the World Health Organization classification, it represents a unique clinical presentation of any subtype of AML, rather than being a specific subtype of AML in its own right. Myeloid sarcoma can precede or occur simultaneously with bone marrow diseases, sometimes as a manifestation during AML relapse or progression of a prior myelodysplastic syndrome or myeloproliferative neoplasm. The sites of isolated GS include the ovary, uterus, epidural region, soft tissues, periosteum, bone, and lymph nodes as well as less commonly the orbit, intestine, and mediastinum, with involvement of the breast being rare. However, several previous articles have reported on this kind of breast tumor (Table 1). ${ }^{1-7}$

The clinical features and imaging characteristics of breast GS are not specific, and it can therefore easily be misdiagnosed as other breast tumors, such as lymphoma, carcinoma, and benign tumors, especially primary tumors with no evidence of medullary disease. ${ }^{8,9}$ Histopathological examination with immunohistochemical tests is currently the gold standard for diagnosis. Although some experts advocate the treatment of localized GS in relapsed acute promyelocytic leukemia with local radiation to the extramedullary disease site, systemic therapy is also usually required for relapsed disease. Herein, we report an uncommon case of breast GS in a patient and review the relevant literature. Written informed consent was obtained from the patient for publication of the case details and any accompanying images.

\section{Case analysis}

A 34-year-old woman attended our hospital complaining of a palpable mass in the superior lateral quadrant of her right breast. Physical examination revealed a painless and 


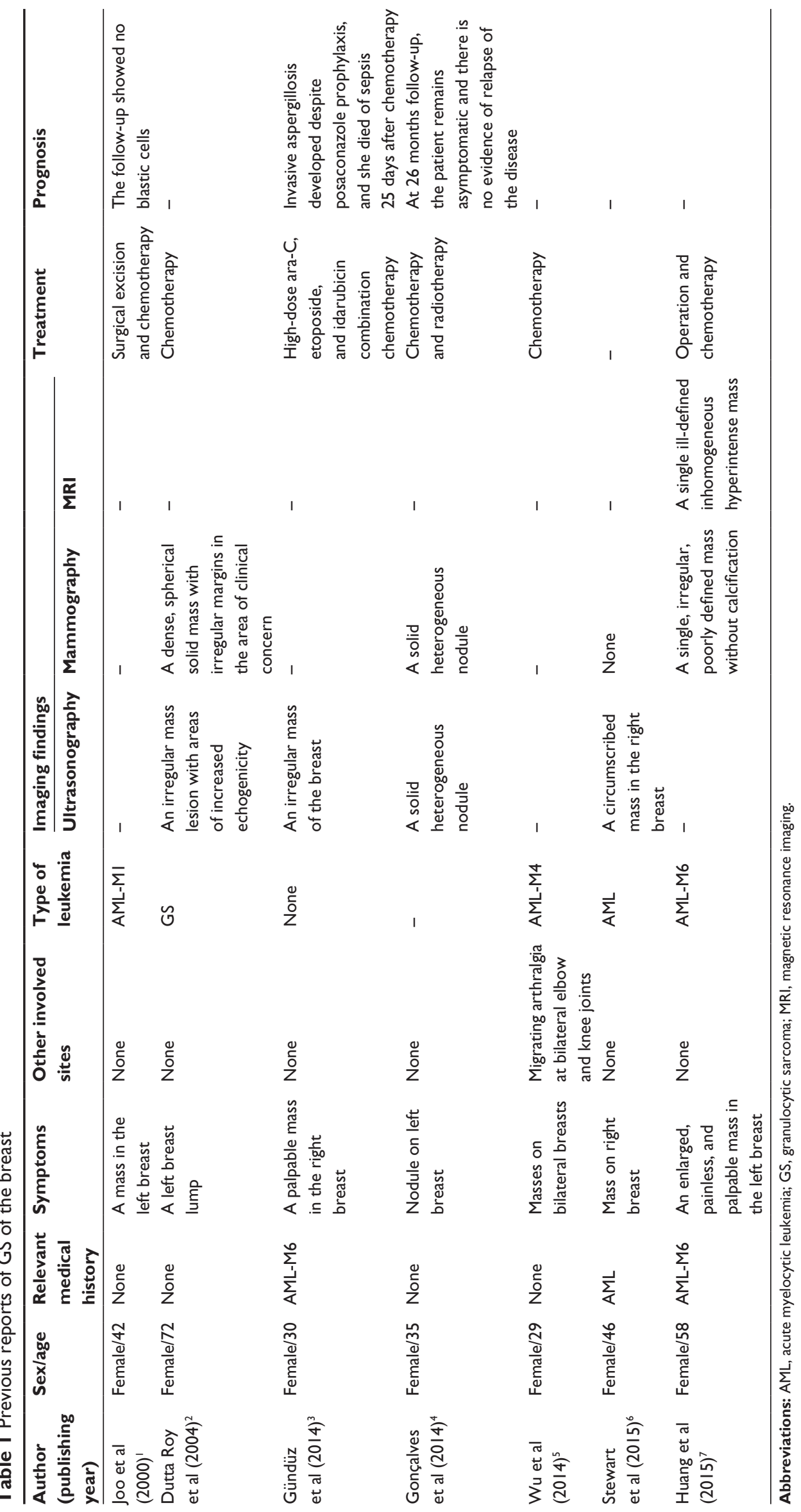


relatively hard, removable mass measuring $\sim 2.6 \times 3.5 \mathrm{~cm}$, with no palpable axillary lymph nodes. Her breast had no nipple discharge, skin ulcers, orange-peel appearance, or nipple retraction. Her medical history included AML (classification: M2) diagnosed 3 years earlier, based on nasosinal spaceoccupying lesions. She underwent six cycles of chemotherapy with cytosine arabinoside and achieved satisfactory disease control and remission, indicated by postchemotherapy bone marrow examination. The patient had no other symptoms or signs and no relevant family history.

A mammogram showed a $\sim 2.5 \times 2.4 \mathrm{~cm}$ round mass with well-defined, irregular margins in the superior lateral quadrant of the right breast, $4.5 \mathrm{~cm}$ from the nipple, with a thick blood vessel passing through the tumor from the chest wall. Another mass was detected in the inferior lateral quadrant of her right breast, $2.1 \mathrm{~cm}$ from the nipple, measuring $\sim 1.4 \times 1.1 \mathrm{~cm}$. Several axillary lymph nodes could be observed on the right side, the largest being $\sim 1.9 \mathrm{~cm}$ in diameter (Figure 1). Breast ultrasound clearly demonstrated a hypoechoic mass measuring $\sim 3.7 \times 2.6 \mathrm{~cm}$ in the superior lateral quadrant of the right breast (Figure 2). The mass was lobulated with an unclear edge, with numerous low-speed bloodstream signals within and close to the mass (resistive index: 0.60). There was another homogenous, hypoechoic mass below the nipple, measuring $\sim 1.8 \times 1.1 \mathrm{~cm}$ in diameter, with a clear edge and an irregular shape. Color Doppler flow imaging showed rich blood supply signals from the surround-

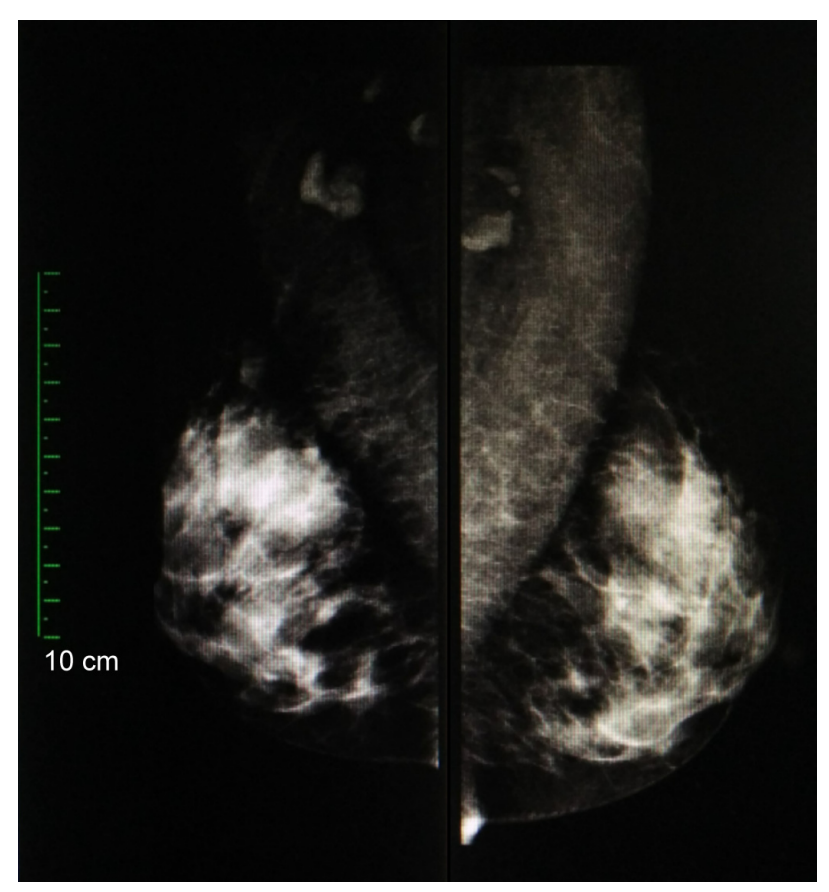

Figure I Mammogram showing a round mass with well-defined, irregular margins in the superior lateral quadrant of the right breast. ing soft tissue. There was a well-defined, hypoechoic nodule $\sim 1.8 \mathrm{~cm}$ in diameter within the right armpit. No other focal masses were observed on the ultrasonographic image.

A complete blood count demonstrated a white blood cell count of $8.07 \times 10^{9} / \mathrm{L}$, a neutrophil count of $5.81 \times 10^{9} / \mathrm{L}$, the hemoglobin levels of $137 \mathrm{~g} / \mathrm{L}$, and a platelet count of $188 \times 10^{9} / \mathrm{L}$. The results of chest radiography and abdominopelvic computed tomography were normal. Fine needle aspiration of the two tumors was performed, and inflammatory cell infiltration and granular calcification with several atypical epithelial cells in the background were identified, suggesting possible malignancy. Considering the differential diagnosis between breast cancer and lymphoma, we performed a bone marrow aspirate examination, which revealed no abnormalities. A lumpectomy was therefore performed under local infiltration anesthesia. The cut surface demonstrated two tumors, well-circumscribed in relation to the adjacent structures. The tumors were $\sim 3 \times 3 \times 2.5 \mathrm{~cm}$ and $2 \times 2 \times 2 \mathrm{~cm}$ and were located $\sim 0.5 \mathrm{~cm}$ apart. Based on the examination of frozen sections, the patient was diagnosed with poorly differentiated malignant tumor.

Postoperative paraffin-based histopathology showed dense myeloid cellular proliferation with breast tissue invasion. Immunohistochemical examination demonstrated that the tumor was strongly positive for myeloperoxidase, CD43, and human leukocyte common antigen (Figure 3); weakly positive for CD20, kappa, lambda, and progesterone receptor; and negative for CD79- $\alpha$, CD3, CD38, CD10, CD5, CD56, AE1/AE3, estrogen receptor, and C-erbB-2. The histological features were consistent with breast GS. The patient was treated with four cycles of consolidation chemotherapy postoperatively $\left(12 \mathrm{mg} / \mathrm{m}^{2}\right.$ idarubicin once a day on days 1 and 2, plus $1 \mathrm{~g} / \mathrm{m}^{2}$ cytarabine per half a day on days 1-3) and achieved remission. She was followed up by bone marrow aspiration and breast Doppler ultrasound examinations every 3 months and remained in good health at the 1.5-year follow-up.

\section{Discussion}

GS is an uncommon extramedullary manifestation of AML that occurs as a consequence of leukemic cell proliferation or migration. Hematopoietic precursor cells divide and differentiate into premature cells, which interact with fibroblasts to promote myeloid metaplasia in many nonhematopoietic tissues. ${ }^{8,10} \mathrm{AML}$ is the most common and typical inducing and predisposing factor for GS. There have been a growing number of reports of GS (Figure 4), mostly from North America, Europe, East Asia, and South Asia (Figure 5). 

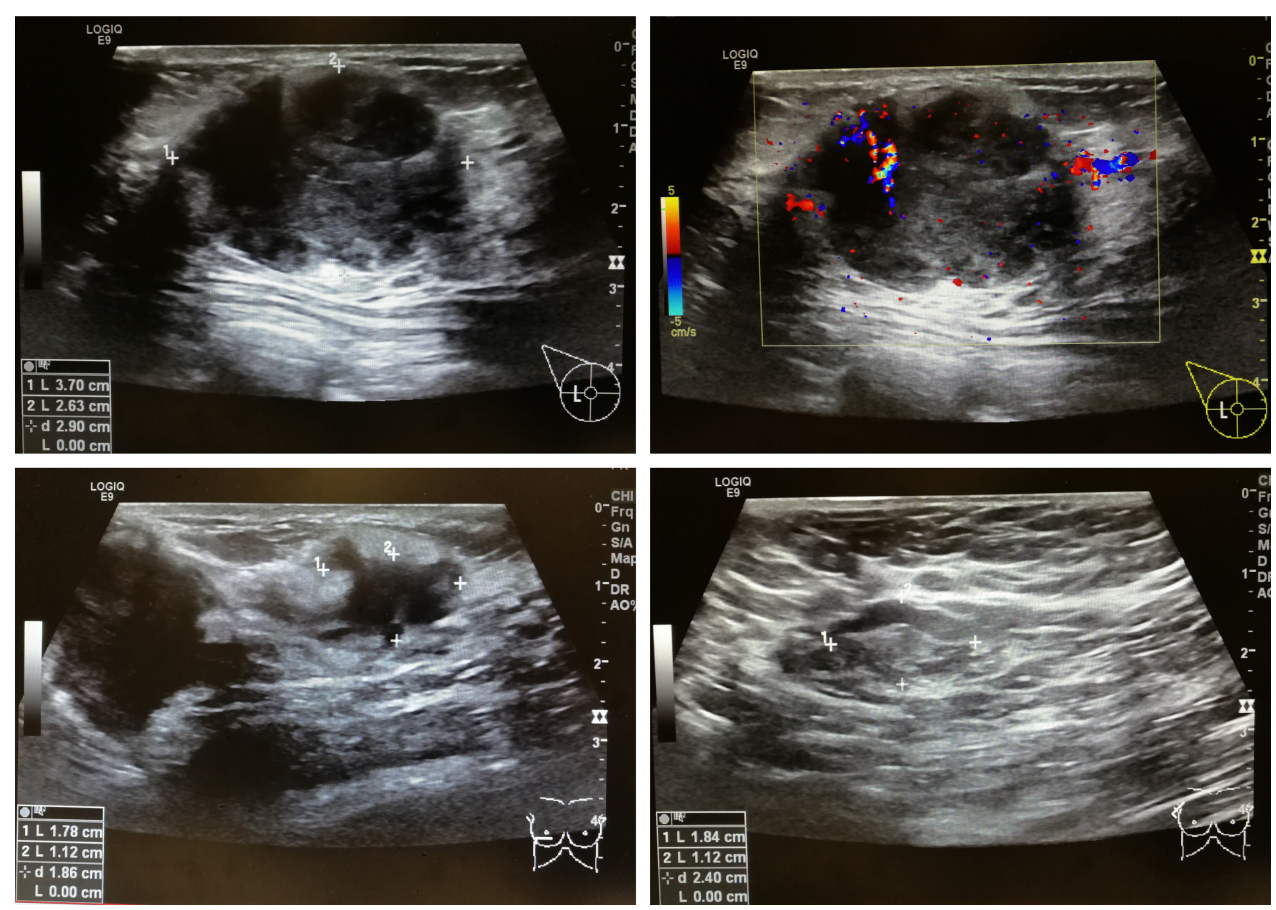

Figure 2 Breast ultrasound demonstrated a hypoechoic mass measuring $\sim 3.7 \times 2.6 \mathrm{~cm}$ in the superior lateral quadrant of the right breast.

The rarity of breast GS means that it is frequently misdiagnosed, most commonly as lymphoma, sarcoma, or breast carcinoma. The clinical findings of GS, including painless and painful palpable breast masses involving unilateral or bilateral breasts, are usually nonspecific, ${ }^{9}$ thus increasing the risk of its clinical misdiagnosis as other primary breast cancers. The breast skin may sometimes be involved, and axillary lymph nodes may be observed, while nipple discharge and retraction are not common. ${ }^{11-13}$ In the current case, an investigation of the patient's medical history and the
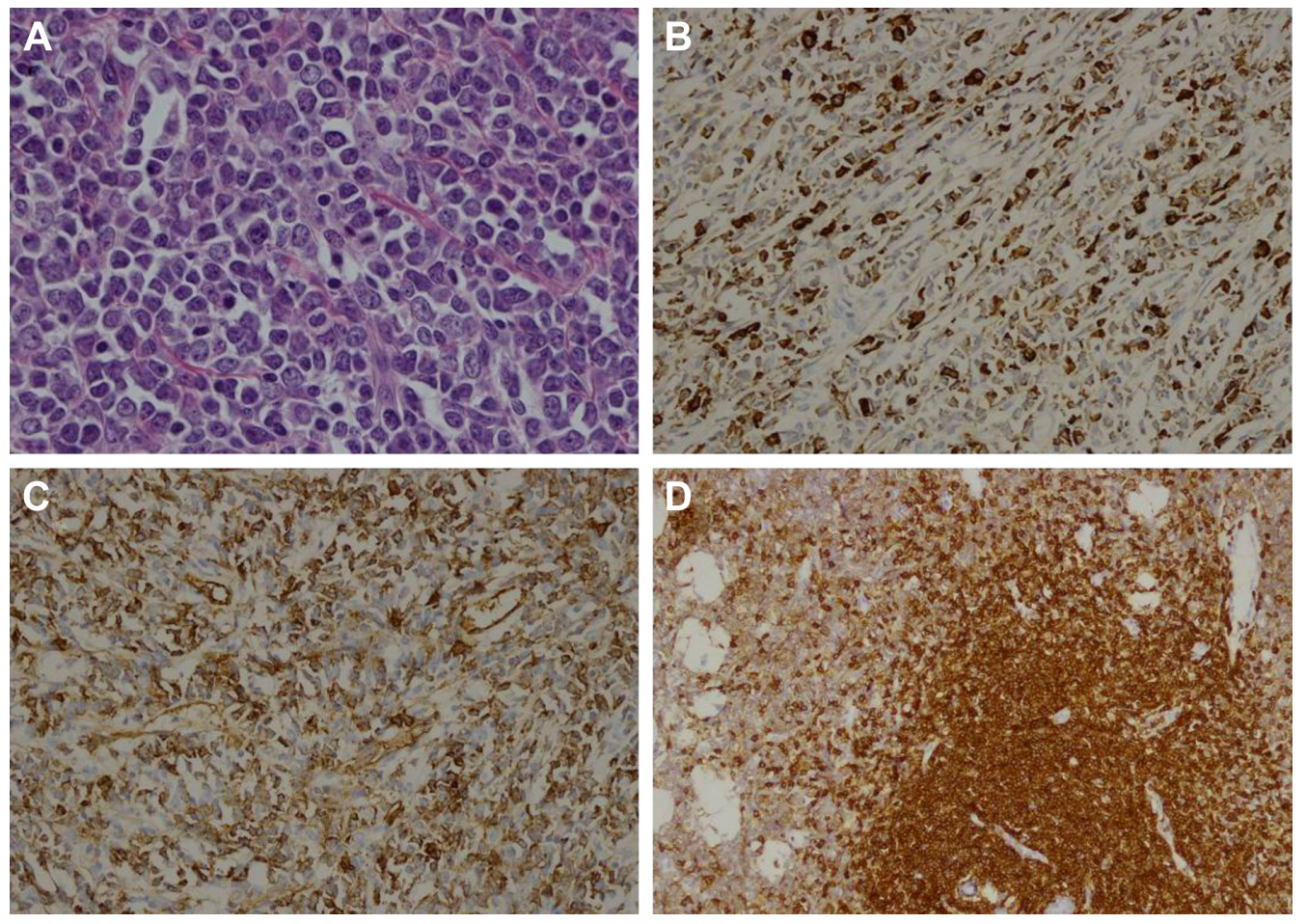

Figure 3 Immunohistochemical results for the resected tumor tissue.

Notes: (A) Hematoxylin-eosin staining; (B) Myeloperoxidase; (C) CD43; and (D) human leukocyte common antigen. Magnification: 20x. 


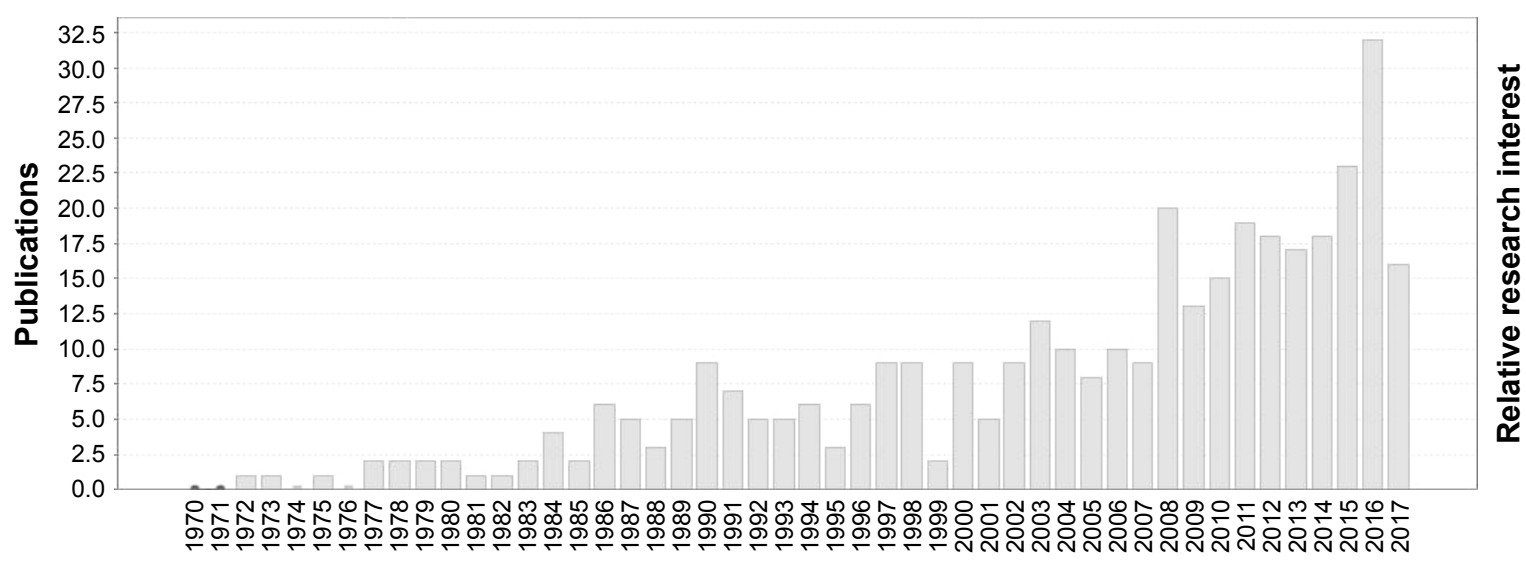

Publications $\rightarrow-$ Relative research interest $\rightarrow$ Relative research interest (smoothed)

Figure 4 Increase in published papers related to granulocytic sarcoma.

results of bone marrow aspiration revealed no abnormalities, making a precise clinical diagnosis difficult.

The results of radiological examinations are also not specific for GS. However, if the patient has a medical history of hematological disease, especially granulocytic leukemia, GS must be kept in mind as a potential differential diagnosis. ${ }^{14}$ Kirubha et al have reported mammogram features, which include tumors (with or without calcification) of variable sizes, with indistinct and hazy borders. ${ }^{15}$ Similarly, ultrasound descriptions of breast GS are rare, though breast GS is generally depicted ultrasonographically as hypoechoic, with spiculated or microlobulated margins. ${ }^{16,17}$ Breast GS may show prominent vascularity on color Doppler ultrasonography. ${ }^{12,18}$ In our case, ultrasound examination revealed two hypoechoic lobulated tumors in the outer upper quadrant of the right breast, with marked vascularity. Magnetic resonance imaging was not employed in the current case, but Thachil et al ${ }^{12}$ reported that T2-weighted coronal images might show GS as hyperintense, heterogeneous, ill-defined, multiple masses relative to the breast parenchyma.

Histopathological examination together with immunohistochemical tests is the gold standard for a final diagnosis of GS, conventionally showing different degrees of myeloid differentiation. ${ }^{9}$ However, the histopathological characteristics are inconsistent, potentially resulting in misdiagnosis or a missed diagnosis, especially in cases of isolated, low-differentiated GS. GS can easily be misdiagnosed as various other tumors, ${ }^{7}$ and breast GS is often confused with infiltrating lobular carcinoma, primary breast sarcoma, Burkitt's lymphoma, and diffuse large B-cell lymphoma. ${ }^{19}$ It is therefore essential to consider GS in the differential diagnosis of breast masses. Immunohistochemical tests, cytogenetic tests, and flow cytometry are useful methods for helping to reduce the misdiagnosis rate, and some molecular

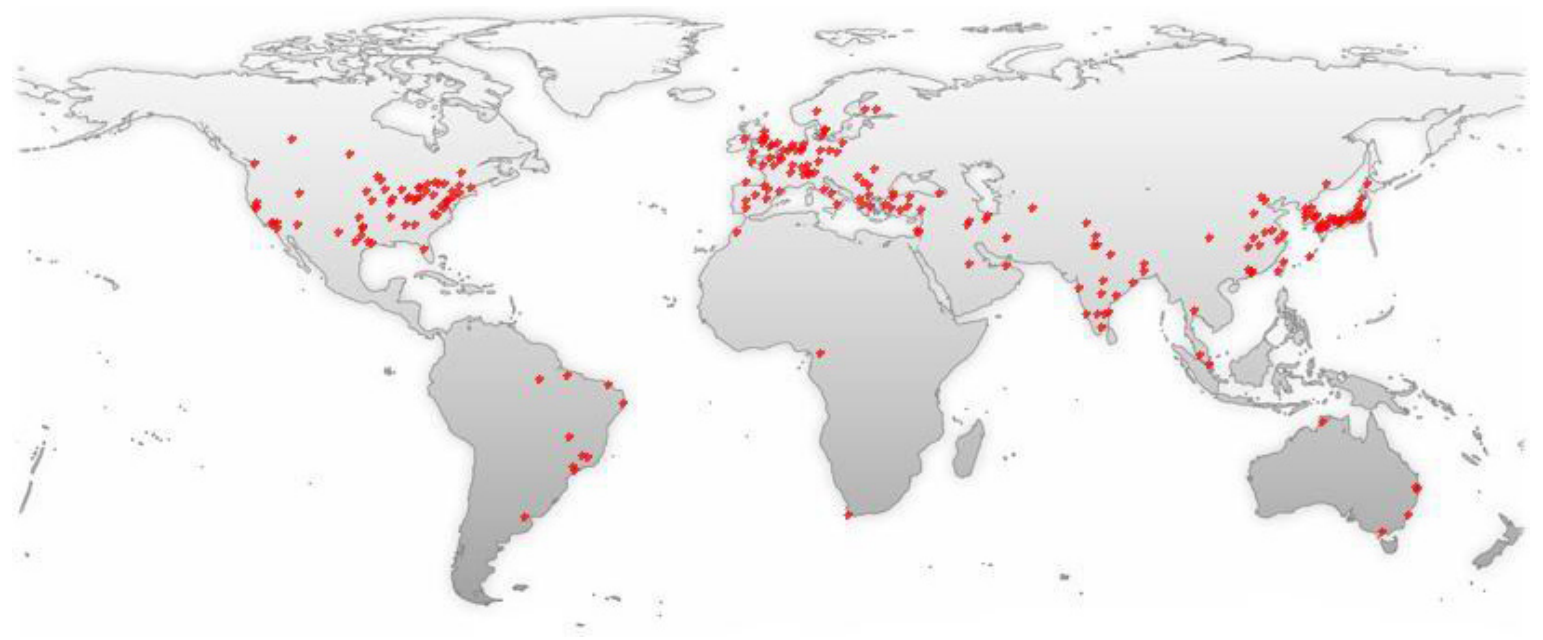

Figure $\mathbf{5}$ Most publications related to granulocytic sarcoma are from North America, Europe, East Asia, and South Asia. 
biomarkers of GS have been identified, including CD117, CD68, lysozyme, nucleophosmin, FLT3-internal tandem duplication, and the biochemical abnormality $\mathrm{t}(8 ; 21)$ inversion. ${ }^{9}$

Hematologists, oncologists, and surgeons have not yet reached a consensus regarding the optimal treatment strategies for breast GS, although lumpectomy or mastectomy with systemic chemotherapy is generally recommended. ${ }^{20}$ Imrie et $\mathrm{al}^{21}$ found that patients with breast GS who received chemotherapy had significantly longer overall survival than those without chemotherapy. In the current case, the patient received postoperative systemic chemotherapy and was in good health at the time of her 1.5-year follow-up.

\section{Conclusion}

GS of the breast is difficult to diagnose, and it is therefore difficult to make clinical decisions regarding its treatment. Although histological results play an important role in its diagnosis, they can also be confusing, especially in poorly differentiated tumors that closely mimic other neoplasms. The present study indicated that lumpectomy combined with systemic chemotherapy could result in a good outcome for patients with GS of the breast. It is therefore essential to include GS among the possible differential diagnoses for breast masses.

\section{Acknowledgments}

We thank Xiangyu Wang, MD, for his contribution to the acquisition and analysis of the data and drafting several parts of the manuscript. We thank Susan Furness, PhD, from Liwen Bianji, Edanz Group China, for editing the English text of a draft of this manuscript.

\section{Disclosure}

The authors report no conflicts of interest in this work.

\section{References}

1. Joo M, Lee HK, Kang YK, Kim JH. Granulocytic sarcoma of the breast preceding acute myelogenous leukemia: a case report. J Korean Med Sci. 2000;15(4):457-459.

OncoTargets and Therapy

\section{Publish your work in this journal}

OncoTargets and Therapy is an international, peer-reviewed, open access journal focusing on the pathological basis of all cancers, potential targets for therapy and treatment protocols employed to improve the management of cancer patients. The journal also focuses on the impact of management programs and new therapeutic agents and protocols on
2. Dutta Roy S, Stafford JS, Scally J, Selvachandran SN. Granulocytic sarcoma of the breast antedating acute myelogenous leukemia. Breast. 2004;13(3):242-246.

3. Gündüz E, Akay MO, Karagülle M, Ak İS. Isolated granulocytic sarcoma of the breast after allogeneic stem cell transplantation: a rare involvement also detected by 18FDG-PET/CT. Turk J Hematol. 2014; 31(1):88-91.

4. Gonçalves J, Louro LV, Ribeiro I, Oliveira Â, Castro C. Radiotherapy for granulocytic sarcoma of the breast - case report and review of the literature. Rep Prac Oncol Radiother. 2014;19(5):343-346.

5. Wu B, Li F, Zou S. MLL-AF9 rearrangement in myeloid sarcomas involving the breast. Ann Hematol. 2014;93(4):709-710.

6. Stewart RL, Dell CM, Samayoa L. Myeloid sarcoma of the breast misdiagnosed as poorly differentiated mammary carcinoma with lobular features. Breast J. 2015;21(2):192-193.

7. Huang XE, Li YJ, Zhou XD. Granulocytic sarcoma of the breast: a case report. Oncol Lett. 2015;10(4):2447-2449.

8. Rizwan M, Islam MM, Rehman ZU. Granulocytic sarcoma of the male breast in acute myeloblastic leukemia with concurrent deletion of $5 \mathrm{q}$ and trisomy 8. Case Rep Hematol. 2012;2012:194312.

9. Fernandes Vieira V, Vo QD, Bouquet de la Joliniere J, Khomsi F, Feki A, Hoogewoud HM. Granulocytic sarcoma presenting as a palpable breast lump. Front Surg. 2016;3:67.

10. Valsamis EM, Glover TE. Granulocytic sarcoma: a rare cause of sciatica. BMJ Case Rep. 2017;2017:bcr2016219009.

11. Surov A, Wienke A, Abbas J. Breast leukemia: an update. Acta Radiol. 2012;53(3):261-266.

12. Thachil J, Richards RM, Copeland G. Granulocytic sarcoma - a rare presentation of a breast lump. Ann R Coll Surg Engl. 2007;89(7): W7-W9.

13. Cunningham I. A clinical review of breast involvement in acute leukemia. Leuk Lymphoma. 2006;47(12):2517-2526.

14. Kinoshita T, Yokokawa M, Yashiro N. Multicentric granulocytic sarcoma of the breast: mammographic, sonographic, and MR findings. Clin Imaging. 2006;30(4):271-274.

15. Kirubha AS, Rachel M, Anburajan M. Automated multimodal segmentation of an abnormal breast mass in mammogram. Proc Inst Mech Eng H. 2016;230(6):516-531.

16. Park JW, Ko KH, Kim EK, Kuzmiak CM, Jung HK. Non-mass breast lesions on ultrasound: final outcomes and predictors of malignancy. Acta Radiol. 2017;58(9):1054-1060.

17. Hasni H, Meah FA, Norlia A, Sharifah NA, Zulfiqar A. Ultrasound in the assessment of the palpable breast mass. Med J Malaysia. 2004; 59(4):486-494.

18. Ahrar K, McLeary MS, Young LW, Masotto M, Rouse GA. Granulocytic sarcoma (chloroma) of the breast in an adolescent patient: ultrasonographic findings. J Ultrasound Med. 1998;17(6):383-384.

19. Fu J, Luo J. Granulocytic sarcoma of the breast in acute myeloid leukemia: two case reports. Oncol Lett. 2014;7(1):145-147.

20. Goncalves J, Louro LV, Ribeiro I, Oliveira A, Castro C. Radiotherapy for granulocytic sarcoma of the breast-case report and review of the literature. Rep Pract Oncol Radiother. 2014;19(5):343-346.

21. Imrie KR, Kovacs MJ, Selby D, et al. Isolated chloroma: the effect of early antileukemic therapy. Ann Intern Med. 1995;123(5):351-353.

patient perspectives such as quality of life, adherence and satisfaction. The manuscript management system is completely online and includes a very quick and fair peer-review system, which is all easy to use. Visit http://www.dovepress.com/testimonials.php to read real quotes from published authors. 